\title{
Geant4 Developments and Applications
}

J. Allison, K. Amako, J. Apostolakis, H. Araujo, P. Arce Dubois, M. Asai, G. Barrand, R. Capra, S. Chauvie, R. Chytracek, G. A. P. Cirrone, G. Cooperman, G. Cosmo, G. Cuttone, G. G. Daquino, M. Donszelmann, M. Dressel, G. Folger, F. Foppiano, J. Generowicz, V. Grichine, S. Guatelli, P. Gumplinger, A. Heikkinen, I. Hrivnacova, A. Howard, S. Incerti, V. Ivanchenko, T. Johnson, F. Jones, T. Koi, R. Kokoulin, M. Kossov, H. Kurashige, V. Lara, S. Larsson, F. Lei, O. Link, F. Longo, M. Maire, A. Mantero, B. Mascialino, I. McLaren, P. Mendez Lorenzo, K. Minamimoto, K. Murakami, P. Nieminen, L. Pandola, S. Parlati, L. Peralta, J. Perl, A. Pfeiffer, M. G. Pia, A. Ribon, P. Rodrigues, G. Russo, S. Sadilov, G. Santin, T. Sasaki, D. Smith, N. Starkov, S. Tanaka, E. Tcherniaev, B. Tomé, A. Trindade, P. Truscott, L. Urban, M. Verderi, A. Walkden, J. P. Wellisch, D. C. Williams, D. Wright, and H. Yoshida

\begin{abstract}
Geant4 is a software toolkit for the simulation of the passage of particles through matter. It is used by a large number of experiments and projects in a variety of application domains, including high energy physics, astrophysics and space science, medical physics and radiation protection. Its functionality and modeling capabilities continue to be extended, while its performance is enhanced. An overview of recent developments in diverse areas of the toolkit is presented. These include performance optimization for complex setups; improvements for the propagation in fields; new options for event biasing; and additions and improvements in geometry, physics processes and interactive capabilities.
\end{abstract}

Index Terms-Electromagnetic interactions, hadronic interactions, object-oriented technology, particle interactions, physics validation, simulation.

\section{INTRODUCTION}

T HE Geant 4 simulation toolkit [1] provides comprehensive detector and physics modeling capabilities embedded in a flexible structure.

Its kernel encompasses tracking; geometry description and navigation; material specification; abstract interfaces to physics processes; management of events; run configuration; stacking for track prioritization; tools for handling the detector response; and interfaces to external frameworks, graphics and user interface systems. Geant 4 physics processes cover diverse interactions over an extended energy range, from optical photons and thermal neutrons to the high energy reactions at the Large Hadron Collider (LHC) and in cosmic ray experiments. Particles tracked include leptons, photons, hadrons and ions. Various implementations of physics processes are offered, providing complementary or alternative modeling approaches. Moreover Geant 4 provides interfaces to enable its users to interact with their application, and save their results. Visualization drivers and interfaces, graphical user interfaces and a flexible framework for persistency are included in the toolkit.

Geant 4 adopts object-oriented technology and follows an iterative-incremental software process [2]. This technology facilitates the extension and refinement of the toolkit, without affecting the existing code used in production mode by many experiments.

Manuscript received November 18, 2005; revised November 30, 2005. Please see Acknowledgment section for author affiliations.

Digital Object Identifier 10.1109/TNS.2006.869826
This paper provides an update of new developments undertaken by the Geant 4 Collaboration to extend the toolkit functionality since the first publication [1], and overviews of validation activities and Geant 4 applications in a variety of experimental domains. The new features described in this paper are publicly available in Geant4 version 7.0, released in December 2004, and following versions.

\section{New Developments IN THE GeANT4 KeRnEL}

The essential kernel capabilities, required for modeling experimental setups, for enabling the use of physics modeling options and for recording particle interactions that occur in the setups, were provided in the first public version of Geant 4 at the end of 1998. Subsequent releases focused on providing a number of refinements, improvements, performance enhancement, and additional developments required by the more sophisticated simulation applications pursued by the experiments. Most of these requirements have been concentrated in three areas of kernel development: run and event management, region dependent production thresholds, and variance reduction.

Improvements in the Geant 4 kernel are not limited to the implementation of new functionality, but also include consolidation and continuous monitoring of its performance. These aspects are particularly important at a stage when Geant 4 is used as a simulation production tool by several experiments. Therefore the most recent releases provided a number of improvements to enhance stability for production, and new tools to identify infrequent problems, which can have significant impact during large scale productions or in some critical applications. Continuous checking of computing time is undertaken in order to monitor CPU performance. Benchmark applications for performance monitoring include simple configurations such as a test beam setup and a use case with a complex magnetic field.

\section{A. Run and Event}

The Run Manager module controls the configuration of a Geant4 setup and of its simulation. The module was re-designed, separating its mandatory kernel functionality into a new class. This refinement enables a user to more easily create a customized run-manager, which fits into a more general software framework, yet is compact and easy to maintain. Moreover the Run Manager now reads primary events which are stored in a high energy physics (HEP) standard of the event 
generator format (HEPMC) [3]. It also handles track vectors directly and can merge different sources of primary particles.

In order to improve the link in the chain from a primary particle and its pre-assigned decay products to the trajectories of resulting tracks and to their associated hits, new hooks for user helper classes were created. These optional helpers can carry user information for objects like a primary vertex, a primary particle, an event or a region. A user can associate information of their choice with each of these objects.

A significant design iteration of the General Particle Source [4] class provided a new formula for converting an integral spectrum to a differential one, better interactivity and other improvements.

\section{B. Region-Dependent Production Thresholds}

One of the most significant enhancements of Geant 4 capabilities is the possibility to define regions in the experimental setup, and to set a different particle production threshold in each $r e$ gion.

In early versions of Geant 4 , the same threshold for producing secondary particles was enforced throughout the experimental setup for each particle type. While this feature ensured consistency of the simulation accuracy, it did not reflect the real-life design of most experiments, which are characterized by detectors of very different precision capabilities (e.g., an inner micro-vertex detector and an outer coarse-grained muon detector).

The capability to group geometrical components into regions has been added to Geant 4 , together with the possibility of associating different behaviors to each region. This new ability allows the simulation accuracy and performance to be optimized according to the characteristics and needs of an experiment. For example, a user can group the geometrical components of a micro-vertex detector into a region and the components of a muon detector into another region, so that different thresholds for producing secondary particles can be assigned to these two regions. The resulting simulation would benefit the accuracy of the high resolution detector, and would save computing time where the coarser detector resolution would not justify tracking the lowest energy particles.

\section{Variance Reduction}

Early versions of Geant 4 offered the possibility of introducing event biasing in a simulation through user code. The Geant 4 kernel offers access to user code through the user action and user initialization base classes. To facilitate the usage of variance reduction techniques, general-purpose biasing methods have been introduced into the toolkit. Many applications, including radiation shielding studies, can profit from this functionality to achieve large gains in time efficiency.

A new Geant4 module provides importance biasing, with splitting and Russian roulette [5]; an importance value is associated with each volume. Either the conventional mass geometry (the one used for physics and tracking) or a dedicated artificial parallel geometry can be used for biasing.

Other biasing capabilities added in recent releases include an implementation of the weight-window method and of the re-
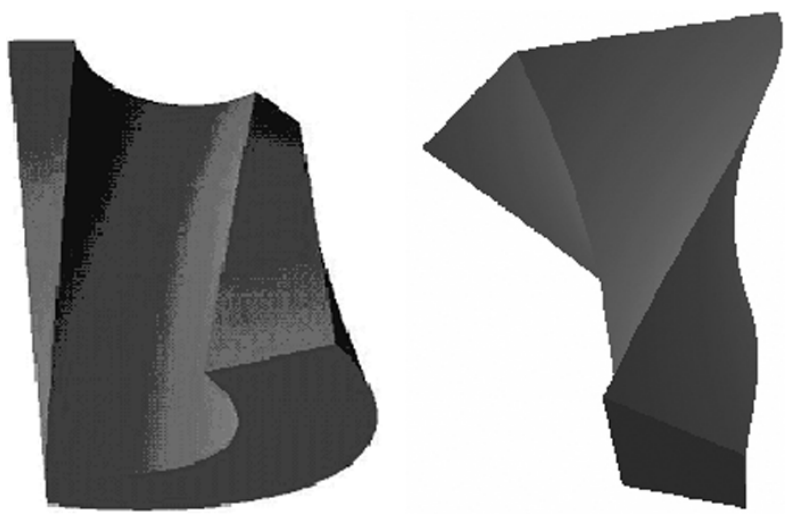

Fig. 1. Ray-tracer view of the Geant4 G4TwistedTubs (left) and G4TwistedTrap (right) solids, with a twist angle of 80 degrees.

lated, but simpler, weight-cutoff method [6]. Leading particle and cross-section biasing are provided for hadronic processes in the corresponding physics package.

\section{IMPROVEMENTS IN DETECTOR MODELING}

\section{A. Geometry}

A significant new feature in Geant 4 geometry is the abstraction of the navigator class G4Navigator. This enables a user to replace or change the Geant 4 navigator, or to add functionality to it. A concurrent design iteration consolidated and simplified the navigator interface.

In early versions of Geant 4 , repeated volumes could be described by replicas representing equal slices of a given volume along a specified axis. All Constructed Solid Geometry (CSG) volumes, including boxes, tubes, cones and polyhedra, can be sliced along an appropriate axis, which is usually a symmetry axis. The existing capabilities of replicas were extended by providing offsets and allowing the creation of replicas of different sizes, through the creation of new division volumes, applied to the mother volume to be sliced.

New shapes have been added to the extensive collection of Geant4 solids; they provide functionality useful for the accurate modeling of detectors currently under construction or being developed for future experiments.

Models to describe twisted solids were introduced in Geant4; the design and algorithmic details can be found in [7]. Stereo mini-jet cells are considered in experiments at a future linear $\mathrm{e}^{+} \mathrm{e}^{-}$collider central tracker such as JLC-CDC [8]. To describe mini-jet cells a new solid, G4TwistedTubs (Fig. 1), was developed. It consists of three different types of bounding surfaces: two end planes, inner and outer hyperboloidal surfaces, and two twisted surfaces. Twisted trapezoids are important components in the Liquid Argon end-cap calorimeter of the ATLAS detector [9]. In Geant 3 a general version of a twisted trapezoid existed, but the implementation put very strong restrictions on its use. In Geant4 no solids were originally available to describe twisted trapezoids. Geant 4 modeling capabilities have been extended to include new G4TwistedBox, G4TwistedTubs, G4TwistedTrd and G4TwistedTrap solids, which are built from two different surfaces: two end planes and four high order surfaces to describe the twisted sides. 
Another new solid, the G4Orb, addresses use cases for a full sphere, allowing for fast performance and enabling the modeling of large spheres.

Improvements to solids focused on a revision of isotropic safety, i.e., the estimated distance to the nearest volume boundary from the current point. The solids affected included some of the CSG solids and their boolean compositions, where rare errors related to the precise estimation of intersections were identified and fixed.

Saving the description of a geometrical setup is a typical requirement of many experiments, which makes it possible to share the same geometry model across various software domains, such as simulation and reconstruction. The geometry description markup language (GDML) [10] and its module for interfacing with Geant 4 have been extended to facilitate a geometrical description based on common tools and standards. A new module enables a user to save a Geant 4 geometry description, which is in memory, by writing it into a text file by extensible markup language (XML) [11]. Support for more solids and for replicas has been added.

In the case of complex experimental setups, it is easy to introduce errors in the geometrical description. In recent releases, new checks at geometry construction time were introduced to ensure that the user creates a geometry that respects Geant 4 geometry rules and constraints, and a new option enables additional checks of the user's geometry during tracking. When using this geometry checking mode, Geant4 provides information on candidate intersections with volume boundaries to help identify underlying problems.

The task of detecting volume overlaps is addressed by specialized tools. These include the DAVID tool [12] for intersecting graphics volumes, and a verification sub-module inside the geometry package. All these tools have adjustable intersection tolerances. The verification sub-module can run different tests and is accessible in any interactive Geant 4 application through user interface commands. Enhancements now allow the user to apply the check by specifying the recursion depth of the geometry setup, a feature of considerable importance, especially for complex structures.

\section{B. Propagation in a Field}

Charged particles in Geant4 are tracked in external electromagnetic fields, and the intersection of their curved trajectories with geometrical boundaries is approximated within a user specified precision.

The module for propagation of charged particles in a field has undergone a performance optimization. A new feature enables a user to attach the same or a different field to a geometrical volume and specialize the integration accuracy parameters for each field. The user can also select, utilizing the track's properties, different values for the accuracy parameters for tracking in a field. This functionality allows a user, for example, to undertake precise tracking for all muons, or for any tracks with energy above a given threshold, while tracking electrons in a calorimeter more coarsely.

\section{PHYSICS EXTENSIONS AND VALIDATION}

The Geant 4 modular architecture makes it an ideal framework into which new components can be integrated and within which new physics models continue to be developed. The extension of Geant 4 physics modeling capabilities is complemented by an extensive validation of the existing models against authoritative reference data and experimental results.

\section{A. Electromagnetic Physics}

A number of developments and improvements have been made in the electromagnetic processes in recent Geant4 releases. These include extensions in physics coverage and modeling capabilities, as well as evolutions of the software to improve the computing performance and to facilitate the usage of the electromagnetic package in experimental applications. The new cuts-per-region functionality, described in Section II.B, affected the implementation of the electromagnetic processes, which evolved to deal with different particle production thresholds in different regions.

The standard electromagnetic package [13] has been redesigned. The new implementation encompasses a model-based design, concentrating the treatment of physics modeling in smaller, dedicated classes. This simplifies maintenance and facilitates extensions and refinements. The new implementation has maintained the user interface unchanged for most use cases. Performance has been improved, in particular for low production thresholds. Refinements include the option to save and retrieve physics tables. This speeds up the startup in execution when several simulation programs run under the same physics configurations, aiding in particular interactive use in setups with many materials. Improvements in multiple scattering, muon and ion ionization, and in simulation of electron-positron pair production by muons were implemented. A new multiple scattering model has been introduced, which can be used also for ions; it provides improved sampling of the tail of the angular distribution and less dependence on the step size.

For optical photons, a new process implements wavelength shifting, and a revision improves the handling of surface properties for material interfaces.

The low energy electromagnetic package [14] addresses especially the requirements of precise simulation, extending Geant4 capabilities below $1 \mathrm{keV}$. Recent developments in low energy physics include high precision models for the angular distribution of Bremsstrahlung photons from electrons below $500 \mathrm{keV}$ [15], new processes for photons, electrons and positrons based on models originally developed for the Penelope [16] Monte Carlo code, a new process for polarized Rayleigh scattering, and the implementation of protons induced X-ray emission (PIXE) [17].

\section{B. Hadronic Physics}

The most recent Geant4 versions provide improvements of previously released hadronic models [18] and further new models. The new developments include theoretical hadronic models for intra-nuclear transport: the first release of the $\mathrm{Bi}$ nary Cascade [19] model and an implementation of the Bertini Cascade [20]. Also available is the chiral invariant phase space 
model (CHIPS) [21], which is the quark-level event generator for the fragmentation of hadronic systems into hadrons. Biasing options for physics processes were also added.

The Binary Cascade was extended to include pion projectiles and light ion reactions and an improved transition [22] to the pre-equilibrium model [23]. The applicability of the implementation of the Bertini Cascade was extended up to $10 \mathrm{GeV}$, and its suitability for isotope production estimation was verified.

The selection of the element undergoing an interaction, in preparation for creating the final state, has been improved. In addition, the choice of an isotope is now made centrally, before calling the models that create the final state. These improvements enable the use of models that can treat specific nuclei, especially those far from the lane of stability.

New models for ion reactions include an implementation of Wilson's abrasion model [24] for ion-induced reactions and electromagnetic dissociation for ion-ion collisions.

New models for the evaporation phase were implemented including an ablation model for use with abrasion and a new implementation broadly similar to the Generalized Evaporation Model (GEM) [25]. The Fermi breakup model has been completely re-written to significantly improve its performance. New, alternative sets of emission probabilities based on those used in the High Energy Transport Code (HETC) [26], and transition probabilities based on those used in the GNASH [26], [27] reaction theory code have been added for the pre-compound model.

A new theory-based coherent-elastic model [28] utilizes preprocessed tabulations for elastic scattering. Also available are new implementations of muon nuclear absorption, an improved fast radioactive decay and alternative GNASH2 transition probabilities in the exciton pre-compound model.

Improvements to cross sections include pion scattering data of Barashenkov [29], which remove existing discontinuities. A legacy problem in high energy $\mathrm{p}-\mathrm{H}$ cross-sections was resolved.

For ion-ion cross-sections, improvements include an implementation of Tripathi's systematics [30] for light ions and parameterizations from Shiver [31], Kox [32] and Shen [33]. Improvements in the scattering term extended its use for nucleon induced reactions up to $8 \mathrm{GeV}$. The S-wave absorption of pions and pion induced reactions up to $1.5 \mathrm{GeV}$ were added to the $\mathrm{Bi}$ nary Cascade model. This energy is the upper limit for pions in this approach, due to current knowledge of strong resonances.

Two forms of biasing were added in the hadronic framework. The first enables leading particle biasing for any reaction, and the second provides cross section biasing for electron-nuclear and gamma-nuclear reactions.

\section{Physics Validation}

A large number of physics observables have been subjected to comparisons between reference data and corresponding simulation data, thus extending the experimental validation of Geant 4 . The validation studies pursued by the Geant 4 Collaboration usually concern basic features of the physics models implemented in the toolkit; their simulated distributions are compared to established references and data in literature. The contributions of

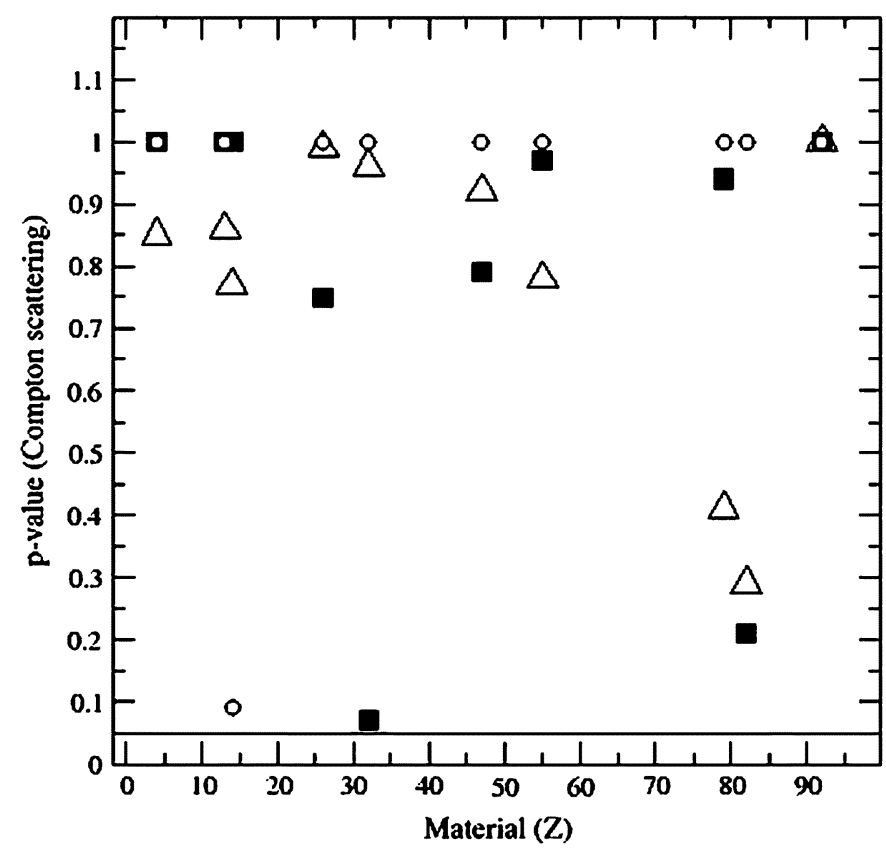

Fig. 2. Validation of Geant4 electromagnetic physics: comparison of various Geant 4 physics models against the NIST data, concerning the cross section of Compton scattering; the symbols represent Geant4 Standard (triangles), Low Energy EPDL (circles) and Low Energy Penelope (squares) models. The continuous line identifies the confidence level set to define the agreement of Geant 4 models with respect to the NIST reference data.

user groups consist mostly of comparisons of full simulation results with experimental data from test beams or on-going experiments, and provide valuable complementary feedback on the accuracy of Geant 4 .

All the electromagnetic models provided by Geant 4 for electrons, photons, protons and $\alpha$ particles have been compared [34] to the National Institute of Standards and Technology (NIST) database [35], [36], which represents a well known, authoritative reference in the field; this database is also adopted in the definition of medical physics protocols. This systematic test involved quantitative comparisons between simulation and reference data, using statistical methods [37]. It confirmed good agreement of all Geant4 electromagnetic models with the NIST reference and highlighted details peculiar to each model, which cause their behavior to differ as a function of the incident particle energy. Fig. 2 shows the comparison of the photon attenuation coefficient obtained with various Geant 4 simulation models against the corresponding NIST reference data. The p-value resulting from the $\chi^{2}$ test represents the probability that the test statistics has a value at least as extreme as that observed, assuming that the null hypothesis (equivalence between Geant 4 simulation and NIST data distributions) is true; $p$-values greater than the confidence level of 0.05 led to the acceptance of the null hypothesis.

Other comprehensive validation studies concerning Geant 4 electromagnetic physics addressed transmission and backscattering distributions [38] and other specific applications of the standard package [39]. Two complementary approaches [40] are adopted for the validation of simulation results in the domain of hadronic physics: one is based on thin target setups with simple geometry, that allow testing single interactions 


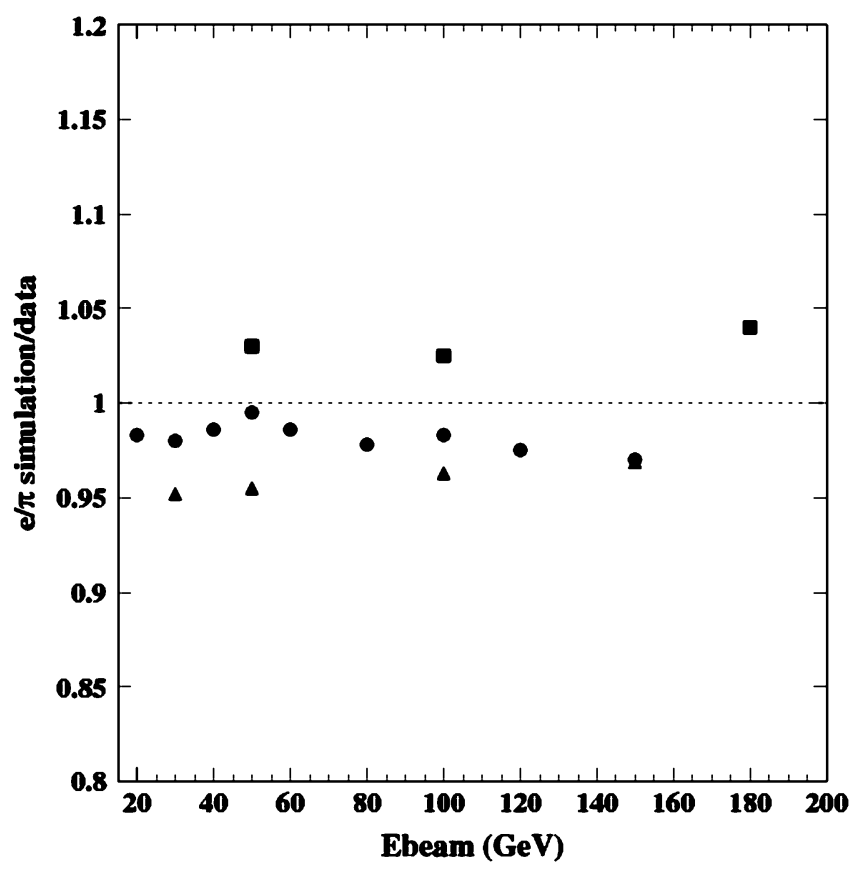

Fig. 3. Ratio between the QGSP simulation and test beam data for the $\mathrm{e} / \pi$ ratio as a function of energy. The symbols represent the ratios for ATLAS HEC Liquid Argon Calorimeter (circles), ATLAS Tile Calorimeter (squares) and CMS Hadronic Calorimeter (triangles). QGSP version 2.7 is used in all the simulations.

or effects in a clean and simple environment; the other relies on calorimeter test beam setups, in which the observables are convolutions of many complex processes and interactions. Fig. 3 shows an example of the comparison of the e $/ \pi$ ratio between a Geant 4 simulation and preliminary experimental data coming from three different calorimeter test beams (ATLAS HEC Liquid Argon Calorimeter [41], ATLAS Tile Calorimeter [42] and CMS Hadronic Calorimeter [43]). In this Geant4 simulation the Physics List which is based on the quark gluon string model (QGSP) is used. More details about the Physics List are described in Section VI.B. As seen in the figure, there is good agreement [44] between simulation and data, at the level of $5 \%$ for the e $/ \pi$ ratio above $20 \mathrm{GeV}$. In Fig. 3, the error bars are not shown because the evaluation of the experimental systematic errors is still in progress to better understand the detector behavior.

\section{ENHANCEMENT OF GEANT4 INTERACTIVE CAPABILITIES}

\section{A. Extensions in Visualization}

Significant work has gone into the HepRep graphics systems [45], which is a generic and hierarchical format for description of graphics representables. Geant 4 can now produce both the earlier HepRep1 format and the newer expanded HepRep2 format. Three different browsers are now available for viewing these files: the WIRED3 HepRep browser [46], the WIRED $J A S$ plugin [47], and the Fox Ruby Event Display FRED [48]. Geant4 HepRep output of detector geometry, particle trajectories, hits and their digitization includes a great deal of useful HepRep attribute information (material names, densities, particle ids, momenta, etc.). All HepRep browsers allow one to view this information by picking on the relevant display

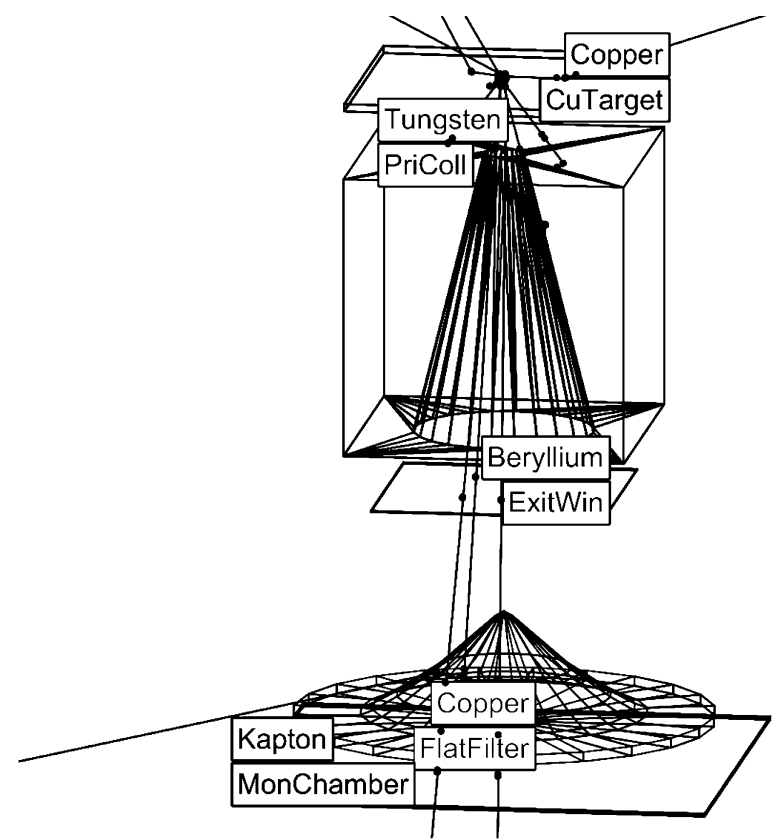

Fig. 4. Geant4 Visualization using the WIRED3D HepRep browser enables a user to label an image with attributes such as material or energy.

objects, and the WIRED3 HepRep browser can cut on these attributes or write any such attributes as visible labels on the display (Fig. 4).

Some improvements in the user interface simplify the sequence of commands for visualization. The flush command which used to be required in most situations is now generally implicit. Commands for end-of-event action and end-of-run action still allow a user to accumulate trajectories and hits from event to event or from run to run with full flexibility.

Improvements have been made to the ASCIITree visualization system, which visualizes the tree structure of a user defined geometry. It can now calculate the mass of the complete geometry tree taking into account daughters down to the depth specified for each physical volume in the current scene. The calculation involves subtracting the mass of that part of the mother which is occupied by each daughter and then adding the mass of the daughter, and so on down the hierarchy.

The OpenGL driver, which previously worked only with Linux, was improved and also runs with the Windows operating system. The OpenInventor driver no longer requires the HepVis graphic library, but instead works with the more widely used ones like Coin3D graphic library [49] or SGI Free Inventor packages.

\section{B. Extensions in User Interface}

Considerable effort was devoted to making Geant4 executable in client-server environments, where, for example, a Geant 4 application running on the remote center can be controlled by a Graphical User Interface (GUI) application running on a user desktop system (Windows, Linux or Mac). This operating mode required the development of Geant4 front-end classes and corresponding client GUI tools. Their design was based on the pre-existing G4UIGAG front-end classes and the Geant4 Adaptive GUI (GAG) tool, implemented in Java. 
The client GUI tools reuse GAG classes to provide users with the same look-and-feel as GAG, but utilize three different modes of communication with the remote Geant 4 application: standard socket connection, SSH connection, and Java remote method invocation (RMI) connection.

The first two modes are implemented in Geant4 Adaptive Interface for Network (GAIN) [50] which re-uses the Java Terminal Application package (JTA) [51]. GAIN guarantees the same level of security as remote execution when using SSH, but provides the same user convenience as GAG.

For the Java RMI connection, the tool uses GAGPlugin that is a plug-in module of JASSimApp, a JAS3 [52] Geant4 Simulation Application [53].

\section{Interface to Data Analysis Systems}

The analysis of simulation results is a typical requirement in real-life experimental applications, as well as an essential component of the physics validation studies carried out by the Geant 4 Collaboration. A Geant 4 application can utilize the analysis system of the user's choice. The advanced examples recently added to the Geant 4 distribution show a recommended design to integrate simulation analysis into a user application: a layer of abstract interfaces decouples the user's code and the Geant 4 toolkit from the implementations of analysis subsystems. To achieve this, the Abstract Interfaces for Data Analysis (AIDA) [54] package, which is an abstract histogram interfaces in HEP and used for example JAS3 or Physics Interface (PI) [55], was chosen for tests and examples. Its main advantage is the ability to change the underlying analysis implementation without modifying any part of the simulation application code.

\section{Using GeAnt4 In Physics ExPERIMENTS}

Geant 4 is a mature simulation tool, used in a wide variety of experimental applications. Recent additions to the toolkit include tools to support its usage in the experimental community, such as examples of real-life applications and sample physics selections for specific experimental use cases. Also added is an example to achieve parallel processing of a simulation based on event parallelism.

\section{A. Application Examples}

The ample choice of physics models and the functionality of a robust kernel enable users to exploit Geant 4 for many diverse applications. The Geant 4 toolkit is complemented by a set of application examples which illustrate its capabilities. Some of these applications-included in the Novice and Extended Examples packages, show the basic usage of the software and its functionality. As a recent extension to the toolkit, a set of $A d$ vanced Examples has been added, demonstrating Geant 4 capabilities in realistic experimental configurations.

Geant 4 Advanced Examples play a significant role in demonstrating the capability of Geant 4 to address experimental requirements specific to various physics domains. They include the simulation of particle detectors (two calorimeters and a Ring Imaging Cherenkov detector), space telescopes for X-ray and $\gamma$-ray astronomy, an underground detector for dark matter searches, electrostatic charging of isolated test masses by galactic cosmic rays, a cosmic ray experiment, and material analysis through a characteristic X-ray fluorescence spectrum. Two simulation applications treat critical situations such as neutron shielding and protection from radioactivity for interplanetary manned missions. Other examples cover various radiotherapy techniques, including brachytherapy, hadrontherapy and intensity modulated radiotherapy. Further advanced examples are under development, in order to extensively document the broad range of applicability of the Geant 4 toolkit.

\section{B. Support for Physics Use Cases}

The Geant 4 toolkit offers a variety of options for physics processes and models over a wide range of energies for electromagnetic and strong interactions. For the same combination of projectile and target at a given energy, there can be several models or processes applicable with different accuracy, strengths and computational cost. It is possible to create numerous configurations of models in order to address the needs of a particular use case.

Making an optimal selection of a set of models among those available can present a daunting learning curve, especially for hadronic interactions. The absence of a unique effective theory of hadronic interactions, which is calculable at relatively low energies, is a significant obstacle. In addition the variety of approaches even at high energies, where scaling formula and applicability of perturbative Quantum Chromodynamics (QCD) enable simulation, provide several competing approaches (chains, diffraction, hadronic clusters, Quark-Gluon strings). The low energy hadronic models, whose approximate validity is often restricted to particular incident particles, target material types, and interaction energies require special efforts from users. By using a consistent, tailored set of models it is possible to address the requirements of a particular use case.

Choosing among the Geant 4 hadronic models is made easier by a number of Physics Lists which are included in the Geant 4 toolkit release. Each Physics List is a complete and consistent collection of models chosen to be appropriate for a given use case. Hadronic use cases relevant to high energy physics applications include calorimeters, trackers and a typical general-purpose detector. At low energy the use cases of neutron dosimetry applications and nucleon penetration shielding are covered. Results already obtained for several use cases and physics list are available, many obtained by users, and provide invaluable reference points and benchmarks.

\section{Running Geant4 as a Parallel Application}

Simulation applications often involve the generation of a large number of events and require significant computing resources. Execution in a parallel mode contributes to adequate simulation statistics in a reduced time frame. The use of job-level parallelism, using independent jobs on farms of computers, is well established and well suited when there is experience in distributing jobs and gathering the results. Yet in other domains different types of parallelism provide an alternative with simpler ways to launch jobs and obtain results.

An extended example shows how to use the task oriented parallel package TOP-C [56] to parallelize Geant4 using event- 
level parallelism [57]. Even though the application actually runs in parallel on distributed computers, it appears to run as a single process with a single Geant4 library, storing and analyzing a single collection of hits. The master process transparently generates events that are processed on slave processes, and whose hits are transparently brought back to the master.

In previous versions, the application writer had to modify the example code to marshal (serialize) the application-defined hits, so they could be transported across the network. The recent extension employs a Marshalgen tool [58], to automate writing the code for marshalling hits. The application writer adds comments (annotations) in the header file for the application-defined hits. The Marshalgen package then automatically generates the required code for marshalling hits.

Running Geant4 as a parallel application has also been demonstrated using the DIANE framework [59], which provides a distributed data analysis environment.

\section{CONCLUSION}

Geant 4 was the first large scale software project to pioneer the adoption of object-oriented technology in particle physics. The choice of this novel technology has enabled the initial development of a powerful toolkit with rich functionality, and its further extension and refinement without affecting the original kernel. Many new features have been added since the first public release of Geant4 in the domains of detector and physics modeling, in response to the requirements of a wide user community. The extension and improvement of Geant 4 physics capabilities, both in the electromagnetic and hadronic domain, represent a continuous effort to improve the simulation accuracy offered to experimental applications over a wide energy range. The software technology has also supported a smooth evolution in the kernel itself, such as the introduction of the novel concept of detector regions. The interactive capabilities of the toolkit have been extended for a more user-friendly approach.

The development of new features is complemented by a parallel program of validation of Geant 4 physics models against experimental data, and by the demonstration of its capabilities in real-life experimental contexts. The results of these associated activities provide important guidance to the users to best exploit rich functionality of Geant 4 .

The Geant 4 Toolkit is nowadays used by a wide scientific community worldwide in diverse experimental domains. Geant4 is used in production by large scale high energy physics experiments as well as in smaller scale detector development projects. It is also employed for accurate simulation in mission critical applications in space science and astrophysics, and is the basis for accurate calculations in medical physics, nuclear medicine and radiation protection.

The development of the Geant 4 Toolkit will continue to be pursued in the future by the Geant 4 Collaboration, in response to the evolving requirements of the wide experimental community using it. Significant effort is also invested in the validation of its physics models.

\section{ACKNOWLEDGMENT}

We express our appreciation for the wealth and depth of feedback provided by many Geant 4 users, which has greatly helped in improving the toolkit and validating it for diverse use cases. The LIP group acknowledges the support under FCT-Portugal, project POCTI/FP/FNU/50 129/2003. The INFN Sezione di Genova group acknowledges the support of the European Space Agency through Contract no. 17696/03/NL/AG and Contract no. $14402 / 00 / \mathrm{NL} / \mathrm{MV}$.

J. Allison and A. Walkden are with the University of Manchester, Manchester M13 9PL, U.K.

K. Amako, K. Murakami, and T. Sasaki are with KEK Tsukuba Ibaraki, Ken 305-0801, Japan (e-mail: Katsuya.Amako@kek.jp).

J. Apostolakis, R. Chytrachek, G. Cosmo, G. G. Daquino, M. Dressel, G. Folger, J. Generowicz, M. Kossov, O. Link, I. McLaren, P. Mendez Lorenzo, A. Pfeiffer, A. Ribon, S. Sadilov, E. Tcherniaev, and J. P. Wellisch are with CERN, Geneva CH-1211, Switzerland.

H. Araujo and A. Howard are with Imperial College, London SW7 2AZ, U.K. P. Arce Dubois is with the University of Cantabria, Santander E-39005, Spain. M. Asai, M. Donszelmann, T. Johnson, T. Koi, J. Perl, D. Smith, and D. Wright are with SLAC, Stanford, CA 94309 USA.

G. Barrand is with LAL, Orsay 91898, France.

R. Capra, S. Guatelli, A. Mantero, B. Mascialino, M. G. Pia, and M. Piergentili are with INFN Sezione di Genova, Genova 16146, Italy.

S. Chauvie is with Azienda Ospedaliera Santa Croce e Carle Cuneo and with INFN Sezione di Torino, Torino 10125, Italy.

G. A. P. Cirrone, G. Cuttone, and G. Russo are with INFN Laboratori Nazionali del Sud, Catania 95123, Italy.

G. Cooperman is with Northeastern University, Boston, MA 02115 USA.

F. Foppiano is with IST Istituto Nazionale per la Ricerca sul Cancro, Genova 16132, Italy.

V. Grichine and N. Starkov are with P.N. Lebedev Institute, Moscow 117924, Russia.

P. Gumplinger and F. Jones are with TRIUMF, Vancouver, BC V6T 2A3 Canada.

I. Hrivnacova is with Institut de Physique Nucleaire, Orsay 91898, France.

A. Heikkinen is with Helsinki Institute of Physics, Helsinki FIN-00014, Finland.

V. N. Ivanchenko is on leave from Budker Institute, Novosibirsk RU-630090, Russia.

$\mathrm{S}$. Incerti is with CENBG, CNRS/IN2P3 - Bordeaux 1 University, Gradignan Cedex F-33175, France.

R. Kokoulin is with the Moscow Engineering Physics Institute, Moscow RU-115 409, Russia.

H. Kurashige is with Kobe University, Kobe 657, Japan.

V. Lara is with Instituto de Fisica Corpuscular, Valencia E-46071, Spain.

S. Larsson is with Karolinska Institutet, Stockholm SE-171 77, Sweden.

F. Lei and P. Truscott are with Qinetiq, Farnborough GU14 OLX, U.K.

F. Longo is with INFN Sezione di Trieste, Trieste 34127, Italy.

M. Maire is with LAPP, Annecy-le-Vieux 74941, France.

K. Minamimoto and H. Yoshida are with Naruto University of Education, Tokushima 772-8502, Japan.

P. Nieminen and G. Santin are with the European Space Agency, Noordwijk 2200 AG, The Netherlands.

L. Pandola and S. Parlati are with INFN Laboratorio Nazionale del Gran Sasso, Assergi 67010, Italy. 
L. Peralta, P. Rodrigues, B. Tomé, and A. Trindade are with LIP, Lisbon P-1000-149, Portugal.

S. Tanaka is with Ritsumeikan University, Kusatsu Shiga-Ken 525-8577, Japan.

L. Urban is with RMKI, Budapest H-1525, Hungary.

M. Verderi is with Ecole Polytechnique, Palaiseau 91128, France.

D. C. Williams is with the University of California at Santa Cruz, Santa Cruz, CA 95064 USA.

\section{REFERENCES}

[1] S. Agostinelli et al., "Geant4: A simulation toolkit," Nucl. Instrum. Meth. A, vol. 506, no. 3, pp. 250-303, 2003.

[2] G. Cosmo, "Software process in Geant4," in Proc. Computing in High Energy and Nuclear Physics, Beijing, China, 2001, pp. 469-472.

[3] M. Dobbs and J. B. Hansen, "The HepMC C++ Monte Carlo event record for high energy physics," Comp. Phys. Comm., vol. 134, no. 1, pp. 41-46, 2001.

[4] P. Truscott et al., "A spacecraft radiation shielding and effects toolkit based on Geant4," in Proc. Computing in High Energy and Nuclear Physics, vol. A123, Padova, Italy, 2000.

[5] M. Dressel, "Geometrical importance sampling in Geant4: From design to verification," CERN-OPEN-2003-048, 2003.

[6] T. Booth, "A Sample Problem for Variance Reduction in MCNP," Los Alamos National Laboratory Report LA-10 363-MS, Oct. 1985.

[7] K. Hoshina, K. Fujii, and O. Nitoh, "Development of a Geant 4 solid for stereo mini-jet cells in a cylindrical drift chamber," Comp. Phys. Comm., vol. 153, no. 3, pp. 373-391, 2003.

[8] “ACFA Linear Collider Working Group,” KEK Report 2001-11, Aug. 2001

[9] A. Jeremie, "The ATLAS liquid argon electromagnetic calorimeter construction status," Nucl. Instrum. Meth. A, vol. 518, no. 1, pp. 28-30, 2004

[10] R. Chytracek, "The geometry description markup language," in Proc. Computing in High Energy and Nuclear Physics, Beijing, China, 2001, pp. 473-476.

[11] W. S. Means and E. R. Harold, XML in a Nutshell, 2nd ed: O'Reilly \& Associates, Inc., 2001.

[12] S. Tanaka, K. Hashimoto, and Y. Sawada, "A visual intersection debugger for modeling Geant 4 detector geometry - DAVID," in Proc. Computing in High Energy and Nuclear Physics, Chicago, IL, 1998.

[13] V. N. Ivanchenko, M. Maire, and L. Urban, "Geant4 Standard electromagnetic package for HEP applications," in Conf. Rec. 2004 IEEE Nuclear Science Symposium, vol. N33-165.

[14] S. Chauvie, G. Depaola, V. Ivanchenko, F. Longo, P. Nieminen, and M. G. Pia, "Geant4 low energy electromagnetic physics," Proc. Computing in High Energy and Nuclear Physics, pp. 337-340, 2001.

[15] P. Rodrigues et al., "Geant4 applications and developments for medical physics experiments," IEEE Trans. Nucl. Sci., vol. 51, no. 4, pp. 1412-1419, Aug. 2004

[16] J. Baró, J. Sempau, J. M. Fernández-Varea, and F. Salvat, "Penelope: An algorithm for Monte Carlo simulation of the penetration and energy loss of electrons and positrons in matter," Nucl. Instr. Meth. B, vol. 100, no. 1, pp. 31-46, 1995

[17] S. Guatelli, A. Mantero, B. Mascialino, P. Nieminen, M. G. Pia, and S. Saliceti, "Geant4 atomic relaxation," in Conf. Rec. 2004 IEEE Nuclear Science Symposium, vol. N44-4.

[18] J. P. Wellisch, "Hadronic shower simulation with Geant4," in Proc. Calorimetry in High Energy Physics, Tucson, AZ, 1997, pp. 522-529.

[19] G. Folger, V. N. Ivanchenko, and J. P. Wellisch, "The binary cascade," Eur. Phys. J. A., vol. 21, pp. 407-417, 2004

[20] A. Heikkinen, N. Stepanov, and J. P. Wellisch, "Bertini intranuclear cascade implementation in Geant4," in Proc. Computing in High Energy and Nuclear Physics, vol. MOMT008, La Jolla, CA, 2003.

[21] P. V. Degtyarenko, M. V. Kossov, and H.-P. Wellisch, "Chiral invariant phase space event generator," Eur. Phys. J. A, vol. 9, pp. 421-424, 2000.

[22] G. Folger and J. P. Wellisch, "The binary cascade," in Proc. Computing in High Energy and Nuclear Physics, vol. ID256, Interlaken, Switzerland, 2004.

[23] V. Lara and J. P. Wellisch, "Preequilibrium and equilibrium decays in Geant4," in Proc. Computing in High Energy and Nuclear Physics, Padova, Italy, 2000, pp. 52-55.
[24] J. W. Wilson et al., "NUCFRG2: An Evaluation of the Semiemprical Nuclear Fragmentation Database," NASA Tech. Paper 3533, 1995.

[25] S. Furihata, "Statistical analysis of light fragment production from medium energy proton-induced reactions," Nucl. Instrum. Meth. B, vol. 171, no. 3, pp. 251-258, 2000.

[26] R. E. Prael and M. Bozoian, "Adaptation of the Multistage Preequilibrium Model for the Monte Carlo Method (I)," Los Alamos National Laboratory Report LA-UR-88-3238, 1988.

[27] E. D. Arthur, "The GNASH Preequilibrium-Statistical Model Code," Los Alamos National Laboratory Report LA-UR-88-382, 1988.

[28] V. L. Korotkikh and N. I. Starkov, "Photoproduction of heavy vector mesons on nuclei with excitation of a discrete level," Sov. Journ. of Nucl. Phys., vol. 37, pp. 610-613, 1983.

[29] V. S. Barashenkov, "Cross sections of interactions of particles and nuclei with nuclei," in JINR, Dubna, 1993.

[30] R. K. Tripathi, F. A. Cucinotta, and J. W. Wilson, "Universal Parameterization of Absorption Cross-Sections-Light Systems," NASA Tech. Paper TP-1999-209726, 1999.

[31] L. Sihver, C. H. Tsao, R. Silberberg, T. Kanai, and A. F. Barghouty, "Total reaction and partial cross section calculations in proton-nucleus $\left(Z_{t}<=26\right)$ and nucleus-nucleus reactions $\left(Z_{p}\right.$ and $\left.Z_{t}<=26\right)$," Phys. Rev. C, vol. 47, no. 3, pp. 1225-1236, 1993.

[32] S. Kox et al., "Trends of total reaction cross sections for heavy ion collisions in the intermediate energy range," Phys. Rev. C, vol. 35, no. 5, pp. 1678-1691, 1987.

[33] S. Wen-qing, W. Bing, F. Jun, Z. Wen-long, Z. Yong-tai, and F. En-pu, "Total reaction cross section for heavy-ion collisions and its relation to the neutron excess degree of freedom," Nucl. Phys. A, vol. 491, no. 1, pp. 130-146, 1989.

[34] K. Amako et al., "Comparison of Geant4 electromagnetic physics models against the NIST reference data," IEEE Trans. Nucl. Sci., vol. 52, no. 4, pp. 910-918, Aug. 2005.

[35] XCOM: Photon Cross Section Database (Version 1.2), M. J. Berger J. H. Hubbell, S. M. Seltzer, J. S. Coursey, and D. S. Zucker (1999). [Online]. Available: url; http://physics.nist.gov/PhysRefData/Xcom/Text/XCOM.html

[36] ESTAR, PSTAR and ASTAR: Computer Programs for Calculating Stopping-Power and Range Tables for Electrons, Protons and Helium Ions (Version 1.2.2), M. J. Berger, J. S. Coursey, and D. S. Zucker. (2000) [Online]. Available: http://physics.nist.gov/PhysRefData/Star/Text/contents.html

[37] G. A. P. Cirrone et al., "A goodness-of-fit statistical toolkit," IEEE Trans. Nucl. Sci., vol. 51, no. 5, pp. 2056-2063, Oct. 2004.

[38] G. A. P. Cirrone et al., "Precision validation of Geant4 electromagnetic physics," in Conf. Rec. 2003 IEEE Nuclear Science Symposium, vol. $\mathrm{N} 23-2$.

[39] V. N. Ivanchenko et al., "Overview and new developments on Geant4 electromagnetic physics," in Proc. Computing in High Energy and Nuclear Physics, vol. 320, Interlaken, Switzerland, 2004.

[40] A. Ribon, "Physics validation of the simulation packages in a LHC-wide effort," in Proc. Computing in High Energy and Nuclear Physics, vol. 493, Interlaken, Switzerland, 2004.

[41] "Liquid Argon Calorimeter Technical Design Report," ATLAS Collaboration, CERN/LHCC/96-41, 1996.

[42] "Tile Calorimeter Technical Design Report," ATLAS Collaboration, CERN/LHCC 96-42, 1996.

[43] "Hadron Calorimeter Technical Design Report," CMS Collaboration, CERN/LHCC/97-31, 1997.

[44] C. Alexa et al., "Geant4 Hadronic Test Beam Validation With Test Beam Data: First Conclusions,", CERN-LCGAPP-2004-10.

[45] HepRep Interface Definition for HEP Event Display Representables: url [Online]. Available: http://www.slac.stanford.edu/ perl/heprep/index.html

[46] WIRED Event Data Visualizer Based on a JAVA Program: url [Online]. Available: http://www.slac.stanford.edu/BFROOT/www/Computing/Graphics/Wired/

[47] WIRED JAS plugin: url [Online]. Available: http://wired.freehep.org/index.html

[48] FRED Fox Ruby Event Display: url [Online]. Available: http://www.fisica.uniud.it/ glast/FRED/

[49] Coin3D Graphic Library: url [Online]. Available: http://www.coin3d.org/

[50] H. Yoshida, "Secure Graphical User Interface for Geant4," in Conf. Rec. 2003 IEEE Nuclear Science Symposium, vol. N43-2.

[51] Java Telnet Application/Applet: url [Online]. Available: http://www.mud.de/se/jta/ 
[52] Java Analysis Studio JAS3: url [Online]. Available: http://jas.freehep.org/

[53] M. Donszelmann et al., "JASSimApp Plugin for JAS3: Interactive Geant4 GUI," in Proc. Computing in High Energy and Nuclear Physics, vol. 399, Interlaken, Switzerland, 2004.

[54] G. Barrand, P. Binko, M. Donszelmann, A. Johnson, and A. Pfeiffer, "Abstract Interfaces for Data Analysis: Component Architecture for Data Analysis Tools,", SLAC-PUB-9409, CERN-IT-2001-013, 2002.

[55] A. Pfeiffer, "Overview of the LCG applications area software projects," in Conf. Rec. 2004 IEEE Nuclear Science Symposium, vol. N38-2.

[56] G. Cooperman, "TOP-C: A task-oriented parallel C interface," in Proc. 5-th International Symposium on High Performance Distributed Computing (HPDC-'96), Syracuse, New York, 1996, pp. 141-150.
[57] G. Cooperman, H. Casanova, J. Hayes, and T. Witzel, "Using TOP-C and AMPIC to port large parallel applications to the computational grid," Future Gener. Comp. Sy., vol. 19, no. 4, pp. 587-596, 2003.

[58] G. Cooperman and V. H. Nguyen, "Marshalgen: Marshaling objects in the presence of polymorphism," in Proc. of the International Conference on Internet Computing (IC'04), Las Vegas, NV, 2004.

[59] J. Moscicki, S. Guatelli, A. Mantero, and M. G. Pia, "Distributed Geant4 simulation in medical and space science applications using DIANE framework and the GRID," Nucl. Phys. B, vol. 125, pp. 327-331, 2003. 